\title{
Center-of-Mass Properties of the Exciton in Quantum Wells
}

\author{
A. Siarkos, E. Runge, and R. Zimmermann \\ Institut für Physik, Humboldt-Universität zu Berlin, Hausvogteiplatz 5-7, 10117 Berlin, FRG
}

(October 22, 2018)

\begin{abstract}
We present high-quality numerical calculations of the exciton center-of-mass dispersion for GaAs/AlGaAs quantum wells of widths in the range $2-20 \mathrm{~nm}$. The $\vec{k} \cdot \vec{p}$-coupling of the heavy- and light-hole bands is fully taken into account. An optimized center-of-mass transformation enhances numerical convergence. We derive an easy-to-use semi-analytical expression for the exciton groundstate mass from an ansatz for the exciton wavefunction at finite momentum. It is checked against the numerical results and found to give very good results. We also show multiband calculations of the exciton groundstate dispersion using a finite-differences scheme in real space, which can be applied to rather general heterostructures.
\end{abstract}

\section{INTRODUCTION}

Excitons dominate the optical properties of low-dimensional semiconductor heterostructures such as quantum wells (QW) and quantum wires. The relative motion of the constituent particles and their center-of-mass (COM) motion determine different characteristics of the optical spectra and exciton kinetics.

The exciton relative motion in QW is well studied and understood. The confinement of the carriers along one or two spatial directions into regions comparable to or smaller than the bulk exciton size enhances the effect of the electron-hole Coulomb interaction. This results in larger binding energies and oscillator strengths and in an increased stability compared to bulk excitons. Therefore, excitons are observed even at room temperature in these structures. The effect of the reduced dimensionality is as a rule much larger on the exciton groundstate than on the excited states.

Details of the excitonic optical spectra of QW related to the COM motion like, e.g., inhomogeneous broadening and Stokes shift between photoluminescence (PL) and absorption are frequently used for structure characterization. These features are influenced by exciton localization and diffusion in the presence of interface or alloy disorder [1]. Optical spectra and their temporal evolution are determined by the exciton formation processes [2] and the subsequent energy and spin [3] relaxation dynamics. Spatially resolved spectroscopy techniques like micro PL and near-field scanning optical microscopy allow direct observation of exciton COM quantization in local potential minima 4 . All these phenomena are intimately related to the exciton COM properties whereby different energy and COM momentum regions of the exciton dispersion are probed in different processes.

In many heterostructure systems of interest like, e.g., GaAs/AlAs, InGaAs/InP and ZnCdSe/ZnSeS, the exciton can be described in the effective-mass approximation (Wannier exciton) due to its small bulk binding energy (e.g., $4 \mathrm{meV}$ for $\mathrm{GaAs}$ ). In this approximation, the degeneracy of the valence bands at the center of the Brillouin zone for materials of cubic or zinc-blende symmetry was first taken into account by Dresselhaus [5]. He also pointed out the absence of a well-defined COM transformation due to this degeneracy. Altarelli and Lipari [6] calculated the exciton COM dispersion for direct- and indirect-gap bulk semiconductors. They demonstrated that the ambiguity in the choice of the COM transformation can be used to achieve formal simplicity or optimal numerical convergence. For bulk GaAs, where the heavy- to light-hole mass ratio is large, the exciton dispersions are found to be strongly anharmonic and show avoided crossings between different branches.

In semiconductor QW, the broken translational symmetry in the growth direction leads to the splitting of heavy and light holes at the $\Gamma$-point, and subsequent formation of heavy- and light-hole excitons. Due to the large holeto-electron mass ratio, the influence of the valence-band mixing on the COM motion is greater than on the relative motion. The exciton dispersions are, thus, strongly non-parabolic. Direct consequences of the exciton dispersion anharmonicity in QW like slow indirect excitonic transitions due to camel-back shaped dispersions [7] have been experimentally observed 8$]$.

The multiband exciton (i.e., with the full coupling of heavy- and light-hole bands taken into account) has been theoretically and numerically thoroughly investigated at vanishing COM momentum $Q$ [9]. The numerical effort for such calculations remains reasonable due to the high symmetry of this point. In contrast, only a few publications on multiband calculations of exciton COM dispersions in QW exist ( 17 19]) since these are very demanding. Methods for improving the numerical accuracy and reducing the effort of such calculations are clearly necessary. Particularly useful would be an easy-to-use approach that gives the main features of the exciton dispersion with at least moderate accuracy. 
The main focus of the present work lies on the exciton groundstate dispersion and its properties. A secondary goal is to study the feasibility of numerical methods for calculating the exciton groundstate dispersion in more complicated structures like $\mathrm{V}$-groove quantum wires [20]. Excitons in $\mathrm{GaAs} / \mathrm{Al}_{0.3} \mathrm{Ga}_{0.7} \mathrm{As}$ symmetric QW are considered (section II). Two different methods for the dispersion calculation are used: (i) the extension for $Q \neq 0$ of the well-known 113. expansion of the exciton in the product space of electron and hole subband states (section II A), and (ii) a finite-differences scheme in real space (section [I B) with a groundstate-adapted Coulomb discretization [19]. Method (i) gives high-quality numerical results but is not feasible, e.g., for quantum wires of complicated geometry. With method (ii) the groundstate exciton dispersion in a V-groove quantum wire is tractable. Its convergence properties are checked here against the results of (i). Results for V-groove quantum wires will be presented elsewhere.

Improving own previous results [19], we address in detail the problem of the choice of the COM transformation and introduce an optimized, groundstate adapted COM transformation that greatly enhances the numerical accuracy and stability of our results (section [II]).

Quasi as a by-product, a semi-analytical expression for the average exciton groundstate mass suitable for exciton localization problems is derived (section [V]). This expression is of great practical importance since it gives for not too wide QW reliable mass values. The only necessary ingredients are the lowest subband dispersion and a good estimate for the groundstate Bohr radius.

Finally, we discuss the results of the exciton dispersion calculations in momentum- (section $\mathrm{VA}$ ) and real-space (section $\mathrm{VB}$ ). The results of our semi-analytical expression for the average exciton groundstate mass are compared to various other mass expressions as well as with the numerical dispersions separately (section $\mathrm{VC}$ ).

\section{THEORETICAL MODEL}

We consider the well-studied system of direct Wannier excitons in a single symmetric $\mathrm{GaAs}_{\mathrm{A}} / \mathrm{Al}_{0.3} \mathrm{Ga}_{0.7} \mathrm{As}$ type I quantum well grown in $\langle 100\rangle$ direction. Many aspects of the presented results can be effortlessly extended to Wannier excitons in other, more general heterostructures.

In the envelope function approximation, the Wannier exciton is described by the Hamilton operator

$$
H=H_{e}\left(\vec{r}_{e}\right)+H_{h}\left(\vec{r}_{h}\right)+V_{\text {Coul }}\left(\vec{r}_{e}-\vec{r}_{h}\right)
$$

$H_{e, h}$ describe the material-dependent bandstructure of the respective particles in the vicinity of the $\Gamma$-point, and $V_{\text {Coul }}$ stands for the attractive Coulomb interaction. We choose the coordinate system as usual with the $z$-axis in growth direction $\langle 100\rangle ; \vec{r}_{e}=\left(x_{e}, y_{e}, z_{e}\right), \vec{r}_{h}=\left(x_{h}, y_{h}, z_{h}\right)$ denote the space coordinates of electron and hole, respectively. For the materials involved, the conduction band is to a good approximation parabolic; anharmonicities in the conduction band arise mainly through the interaction with the light and split-off valence bands which is small due to the relatively large band gaps. The valence band is adequately described by the Luttinger Hamiltonian 21 . in the axial approximation [9,22, which takes into account explicitly the coupling of the heavy- and light-hole bands $\left(\Gamma_{8}^{v}\right)$ but suppresses warping. The coupling to the split-off $\left(\Gamma_{7}^{v}\right)$ band can be safely neglected for subband states with energies up to approximately $50 \mathrm{meV}$ from the band edge because of the relatively large energy separation. We neglect the effect of the different dielectric constants (no image charge effects) $\sqrt[12]{12}, 15,23$, and also all effects that lead to a small spin-splitting like lack of inversion symmetry of the bulk material 14 or the interfaces 24 as well as the exchange part of the Coulomb interaction [17. The electron spin is irrelevant and will be given a fixed value of $+1 / 2$ in the present work. The quantization axis of the electron spin and of the hole angular momentum $J$ is taken along the growth direction, and we use for the valence band edge states the same convention as in Ref. 25.

The Hamilton operator $H$, Eq. (11), acts within these approximations on a four-component envelope function in the product basis of the conduction and valence band edge states $\left\{\left|\frac{3}{2} m_{J}\right\rangle_{v}\left|\frac{1}{2}+\frac{1}{2}\right\rangle_{c}\right\}$, where the hole-spin projection attains values of $m_{J}=+\frac{3}{2},+\frac{1}{2},-\frac{1}{2},-\frac{3}{2}$ :

$$
\begin{gathered}
H_{e}=\left(-\frac{\hbar^{2}}{2 m_{e}}\left(\partial_{x_{e}}^{2}+\partial_{y_{e}}^{2}+\partial_{z_{e}}^{2}\right)+V_{c}\right) I \\
H_{h}=-\frac{\hbar^{2}}{2 m_{0}}\left(\begin{array}{cccc}
\mathcal{P}+\mathcal{Q} & \mathcal{L} & \mathcal{M} & 0 \\
\mathcal{L}^{\dagger} & \mathcal{P}-\mathcal{Q} & 0 & \mathcal{M} \\
\mathcal{M}^{\dagger} & 0 & \mathcal{P}-\mathcal{Q} & -\mathcal{L} \\
0 & \mathcal{M}^{\dagger} & -\mathcal{L}^{\dagger} & \mathcal{P}+\mathcal{Q}
\end{array}\right)+V_{v} I
\end{gathered}
$$


with

$$
\begin{aligned}
& \mathcal{P}=\gamma_{1}\left(\partial_{x_{h}}^{2}+\partial_{y_{h}}^{2}+\partial_{z_{h}}^{2}\right) \quad \mathcal{Q}=\gamma_{2}\left(\partial_{x_{h}}^{2}+\partial_{y_{h}}^{2}-2 \partial_{z_{h}}^{2}\right) \\
& \mathcal{L}=-i 2 \sqrt{3} \gamma_{3}\left(\partial_{x_{h}}-i \partial_{y_{h}}\right) \partial_{z_{h}} \quad \mathcal{M}=\sqrt{3} \frac{\gamma_{2}+\gamma_{3}}{2}\left(\partial_{x_{h}}^{2}-\partial_{y_{h}}^{2}-i 2 \partial_{x_{h} y_{h}}^{2}\right)
\end{aligned}
$$

and

$$
V_{\text {Coul }}\left(\vec{r}_{e}-\vec{r}_{h}\right)=-\frac{e^{2}}{\epsilon} \frac{1}{\left|\vec{r}_{e}-\vec{r}_{h}\right|} I
$$

$I$ is the $4 \times 4$ unity matrix. The material parameters $\gamma_{1}\left(z_{h}\right), \gamma_{2}\left(z_{h}\right), \gamma_{3}\left(z_{h}\right)$ as well as the offsets $V_{v}\left(z_{h}\right), V_{c}\left(z_{e}\right)$ are piecewise constant functions of $z_{e}, z_{h}$. To ensure that the kinetic operators remain Hermitian in the presence of interfaces, we use the symmetric substitutions

$$
\gamma \partial_{i} \rightarrow\left(\partial_{i} \gamma+\gamma \partial_{i}\right) / 2, \gamma \partial_{i j}^{2} \rightarrow\left(\partial_{i} \gamma \partial_{j}+\partial_{j} \gamma \partial_{i}\right) / 2, \quad i=x, y, z .
$$

The in-plane COM momentum $\vec{Q}=-i \hbar\left(\vec{\nabla}_{e_{\|}}+\vec{\nabla}_{h_{\|}}\right)$is a constant of motion because the interaction term (4) depends only on the relative distance of the two particles. Reflection with respect to the central $x y$ plane, $\sigma_{x y}$, is also a symmetry element for symmetric QW. Consequently, the exciton can be characterized by the parity $P= \pm 1$. Then, the wavefunction factorizes into

$$
\Psi^{\vec{Q} ; P a}\left(\vec{r}_{e}, \vec{r}_{h}\right)=\frac{e^{-i \vec{Q} \cdot \vec{R}}}{2 \pi} \sum_{m_{J}} \Psi_{m_{J}}^{\vec{Q} ; P a}\left(z_{e}, z_{h}, \vec{\rho}\right)\left|\frac{3}{2} m_{J}\right\rangle_{v}\left|\frac{1}{2} \frac{1}{2}\right\rangle_{c}
$$

where $\vec{\rho}=\vec{r}_{e_{\|}}-\vec{r}_{h_{\|}}$is the in-plane particle distance, $\vec{R}$ the COM space coordinate canonically conjugate to $\vec{Q}$, and $a$ stands for the remaining quantum numbers related to the relative motion of the exciton.

The COM space coordinate $\vec{R}$ in $(6)$ is not unambiguously defined because of the anharmonic dispersions of the constituent particles [5]. The COM transformation must be linear in order to preserve the canonical commutation relations of space and momentum operators, and it has in general the form

$$
\vec{R}=\boldsymbol{\beta} \vec{r}_{e_{\|}}+(\mathbf{1}-\boldsymbol{\beta}) \vec{r}_{h_{\|}}, \quad \vec{k}=-i \hbar\left((\mathbf{1}-\boldsymbol{\beta}) \vec{\nabla}_{e_{\|}}-\boldsymbol{\beta} \vec{\nabla}_{h_{\|}}\right) .
$$

In the parabolic case, the free parameter $\boldsymbol{\beta}$ is taken as the scalar

$$
\beta_{\text {parab }}=\frac{m_{e}}{m_{e}+m_{h}}
$$

in order that relative and COM motion completely decouple. For bulk excitons, $\boldsymbol{\beta}$ has been considered in the literature as a scalar, a tensor in real space [6, or even a spinor 26. We will return later on to the problem of an appropriate choice for the COM coordinate $\vec{R}$.

Taking into account the electron spin degeneracy, each exciton state is at least fourfold degenerate. It can be shown in a similar way as has been done for the hole subband states in Ref. 25] that the operator $R_{\pi} T$, with the rotation by $\pi$ about the $z$ axis, $R_{\pi}$, and time-reversal, $T$, transforms between the degenerate states of different parity and opposite electron spin. If one combines this operator with the Pauli matrix $\sigma_{y}^{e}$, which flips only the electron spin, we have apart from an overall phase:

$$
\begin{aligned}
\Psi^{\vec{Q} ;-P a}\left(\vec{r}_{e}, \vec{r}_{h}\right) & =\left(\sigma_{y}^{e} R_{\pi} T\right) \Psi^{\vec{Q} ; P a}\left(\vec{r}_{e}, \vec{r}_{h}\right) \\
& =\frac{e^{-i \vec{Q} \cdot \vec{R}}}{2 \pi} \sum_{m_{J}} \Psi_{m_{J}}^{\vec{Q} ; P a^{*}}\left(z_{e}, z_{h},-\vec{\rho}\right)\left|\frac{3}{2}-m_{J}\right\rangle_{v}\left|\frac{1}{2}+\frac{1}{2}\right\rangle_{c} .
\end{aligned}
$$

Comparing (6) with (9), we find:

$$
\Psi_{m_{J}}^{\vec{Q} ;-P a}\left(z_{e}, z_{h}, \vec{\rho}\right)=\Psi_{-m_{J}}^{\vec{Q} ; P a^{*}}\left(z_{e}, z_{h},-\vec{\rho}\right) .
$$

That is, the (degenerate) state of reversed parity is obtained by inverting the order of the spin components of the exciton envelope, complex conjugating, and changing the sign of the in-plane relative coordinate. Thus changing the multiband exciton parity with fixed electron spin in symmetric QW corresponds to flipping the hole spin in the single-band exciton case. In the axial approximation and for $Q=0$, the different angular momentum components 
decouple [22], and changing the sign of $\vec{\rho}$ in Eq. (10) just changes the sign of two spin components leaving the other two unchanged; this holds no longer at $Q \neq 0$.

We have solved the eigenvalue problem (ii) in two ways which will be discussed in turn: (i) in $\vec{k}$-space, expanding Eq. (6) in the product space of the electron and hole subband states [10], and (ii) in real space, using a finitedifferences scheme. The first method gives very accurate results and is used to reveal the main features of the exciton dispersion. The second method is only suitable for the groundstate dispersion but promises to be feasible for more general structures. It is validated by comparing its results with the ones from the first method.

\section{A. Solution in $\vec{k}$-space}

As a first step, we calculate the single-particle subband states and their dispersions

$$
\begin{aligned}
& H_{e}\left|n_{e} \vec{k}_{e} ; \pm \frac{1}{2}\right\rangle=\mathcal{E}_{n_{e}}\left(k_{e}\right)\left|n_{e} \vec{k}_{e} ; \pm \frac{1}{2}\right\rangle \\
& H_{h}\left|n_{h} \vec{k}_{h} ; p_{h}\right\rangle=\mathcal{E}_{n_{h}}\left(k_{h}\right)\left|n_{h} \vec{k}_{h} ; p_{h}\right\rangle
\end{aligned}
$$

using a transfer-matrix method as in [27]. The respective solutions in the axial approximation are of the form

$$
\begin{gathered}
\left|n_{e} \vec{k}_{e} ; \pm \frac{1}{2}\right\rangle=\frac{e^{i \vec{k}_{e} \cdot \vec{r}_{e} \|}}{2 \pi} e^{i\left( \pm \frac{1}{2}\right) \theta_{e}} \xi_{n_{e}}\left(z_{e}\right)\left|\frac{1}{2} \pm \frac{1}{2}\right\rangle_{c}, \\
\left|n_{h} \vec{k}_{h} ; p_{h}= \pm 1\right\rangle=\frac{e^{i \vec{k}_{h} \cdot \vec{r}_{h}} \|}{2 \pi} \sum_{m_{J}} e^{i m_{J} \theta_{h}} \xi_{n_{h}, p_{h}, k_{h}}^{m_{J}}\left(z_{h}\right)\left|\frac{3}{2} m_{J}\right\rangle_{v} .
\end{gathered}
$$

In Eqs. (11,12) $n_{e, h}$ denote the subband indices, $\vec{k}_{e, h}=\left(k_{e, h}, \theta_{e, h}\right)$ the respective in-plane wavevectors in polar coordinates and $p_{h}$ the hole parity under $\sigma_{x y}$ [25].

In a second step, the exciton wavefunction for a given COM momentum $\vec{Q}$ is expanded into

$$
\Psi^{\vec{Q} ; P a}\left(\vec{r}_{e}, \vec{r}_{h}\right)=\sum_{n_{e} n_{h}} \int d \vec{k} \varphi_{n_{e} n_{h}}^{\vec{Q} ; a}(\vec{k})\left|n_{e} \vec{k}_{e} ;+\frac{1}{2}\right\rangle_{c}\left|n_{h} \vec{k}_{h} ; p_{h}\right\rangle_{v}
$$

with subband states of the two particles combined in such a way that the resulting exciton state has the required parity $P$ and total momentum $\vec{Q}$ :

$$
\vec{k}_{e}=\vec{k}+\boldsymbol{\beta} \vec{Q}, \quad \vec{k}_{h}=\vec{k}-(\mathbf{1}-\boldsymbol{\beta}) \vec{Q}, \quad P=p_{h} \cdot(-1)^{n_{e}+1} .
$$

The last relation reflects that the conduction subband envelopes are even (odd) for odd (even) subband index. Fixing exciton parity $P$ and electron spin eliminates any degeneracy at $\vec{Q} \neq 0$.

With the expansion (13) and the relations (14), the exciton Schrödinger equation takes the form

$$
\left(\mathcal{E}_{n_{e}}\left(\vec{k}_{e}\right)+\mathcal{E}_{n_{h}}\left(\vec{k}_{h}\right)-E_{a}^{X}(\vec{Q})\right) \varphi_{n_{e} n_{h}}^{\vec{Q} ; a}(\vec{k})+\sum_{n_{e}^{\prime} n_{h}^{\prime}} \int d \vec{k}^{\prime} V_{\substack{n_{e}^{\prime} n_{h}^{\prime} \\ n_{e} n_{h}}}^{\vec{Q}}\left(\vec{k}, \vec{k}^{\prime}\right) \varphi_{n_{e}^{\prime} n_{h}^{\prime}}^{\vec{Q} ; a}\left(\vec{k}^{\prime}\right)=0
$$

where $E_{a}^{X}(\vec{Q})$ denotes the energy dispersion of the exciton state. The interaction

$$
V_{\substack{n_{e}^{\prime} n_{h}^{\prime} \\ n_{e} n_{h}}}^{\vec{Q}}\left(\vec{k}, \vec{k}^{\prime}\right)=-\frac{1}{2 \pi} \frac{e^{2}}{\epsilon} \frac{1}{\left|\vec{k}-\vec{k}^{\prime}\right|} \mathcal{F}_{\substack{n_{e}^{\prime} n_{h}^{\prime} \\ n_{e} n_{h}}}^{\vec{Q}}\left(\vec{k}, \vec{k}^{\prime}\right)
$$

is the in-plane 2D Fourier transform of the 3D Coulomb potential modified due to the confinement in $z$-direction. The latter is expressed through form factors [with Eq. (14)]

$$
\underset{\substack{n_{e}^{\prime} n_{h}^{\prime} \\ n_{e} n_{h}}}{\vec{Q}}\left(\vec{k}, \vec{k}^{\prime}\right)=\sum_{m_{J}} \iint d z_{e} d z_{h} e^{-\left|\vec{k}-\vec{k}^{\prime}\right|\left|z_{e}-z_{h}\right|} \xi_{n_{e}}^{*}\left(z_{e}\right) \xi_{n_{h}, \vec{k}_{h}}^{m_{J}{ }^{*}}\left(z_{h}\right) \xi_{n_{e}^{\prime}}\left(z_{e}\right) \xi_{n_{h}^{\prime}, \vec{k}_{h}^{\prime}}^{m_{J}}\left(z_{h}\right) .
$$

The above integrals are calculated analytically since the subband states obtained with the transfer matrix method are combinations of exponential and trigonometric functions. 
The integrable singularity of the Coulomb potential (16) at $\vec{k}=\vec{k}^{\prime}$ is taken care of by adding and subtracting in Eq. (15) the analytically integrable term

$$
C\left(\vec{k}, \vec{k}^{\prime}\right)=\frac{e^{2}}{\epsilon} \frac{1}{2 \pi}\left(\frac{1}{\left|\vec{k}-\vec{k}^{\prime}\right|}-\frac{1}{\left.\max \left(k, k^{\prime}\right)\right)}\right) .
$$

This gives a smooth "corrected" potential (16) of small absolute magnitude.

To take benefit of the axial approximation, the exciton envelope and the form factors are expanded into 2D angular momentum eigenstates $\exp (i \ell \theta)$. The angular momentum $\ell$ is chosen for every subband combination such that $\ell=0$ corresponds to the respective $s$-like exciton [13] at $Q=0$.

The resulting set of coupled one-dimensional integral equations is solved numerically for various values of the COM momentum $Q$. Results will be presented in section $\square$.

\section{B. Solution in real space}

We demonstrated in Ref. [19] that calculations of the multiband-exciton groundstate dispersion are also feasible with a finite-differences scheme in real space. This method is conceptually simple: the Schrödinger equation corresponding to Eqs. (1) -(14),(6) leads to a system of four (number of spin components) coupled partial differential equations in the four dimensional space $\left(\vec{\rho}, z_{e}, z_{h}\right)$. The resulting eigenvalue problem involves a large sparse complex Hermitian matrix with a substantial number, 44 , of nonzero off-diagonals. In contrast to the $\vec{k}$-space approach, the method can, in principle, be applied effortlessly to very general heterostructures, like quantum wires and quantum dots. The main drawback is the need for huge amounts of computer memory. Indeed, the dimension of the matrix to be diagonalized scales with the fourth power of the number of grid points per spatial dimension. The most dense grid we used led to a matrix of dimension $N_{D}=4 \times 31 \times 31 \times 61 \times 61=1.4 \cdot 10^{7}$ ( 4 is the number of spin components).

We used the ARPACK [28] package to calculate a few eigenvalues and eigenvectors at the lower end of the spectrum. ARPACK is an efficient implementation 29] of the Implicitly Restarted Arnoldi Method that can be viewed as a synthesis of the Arnoldi/Lanczos process with the Implicitly Shifted QR algorithm. Storage of the (nonzero) matrix elements is not required, only a matrix-vector multiplication utility is needed. Multiple eigenvalues, as they occur in our case, offer no additional problems. However, if one needs all the members of a multiplet the iteration subspace has to be chosen large enough. We find that an iteration subspace of five times the number of the requested eigenvalues (rather than the proposed factor of two [28]) is usually sufficient. This matter was of no concern for our problem, since using the symmetry considerations, Eq. (10), we can derive from a calculated state also the second one of the doublet. For the largest matrices, we used a factor of three as a compromise between memory demand and CPU time usage.

The matrix resulting from the discretization is highly structured. For minimizing the memory costs and still making full use of the vector registers, we construct the matrix-vector product using auxiliary, much smaller matrices.

Since memory is critical, it is crucial for any real-space approach to optimize the convergence of the relevant quantities with the mesh size. On the one hand, we optimize the COM transformation, as will be discussed in the next section, thereby improving the handling of the kinetic terms. On the other hand, we use a groundstate-adapted discretization of the Coulomb potential [30] which is discussed in more detail in the Appendix. The idea behind this approach is to extract the discretized interaction from a reference system that has the same interaction but a simple kinetic term, and whose groundstate is known analytically. If the groundstate of the reference system is similar enough to the one of the real system, good convergence is expected.

Calculations on a parallel-vector machine of type CRAYJ932 reached performances of 140MFlops/CPU, the peak performance of the limiting BLAS routine being 185MFlops/CPU.

\section{OPTIMIZED CENTER-OF-MASS TRANSFORMATION}

We return to the ambiguity in the COM transformation (7) which is expressed in the freedom to choose $\boldsymbol{\beta}$. The relevance of $\boldsymbol{\beta}$ for accelerating numerical convergence in dispersion calculations was realized quite early for bulk excitons in [6], where a scalar $\beta$ was optimized in a trial and error procedure. However, there has been no other algorithm to take advantage of this freedom until recently [19]. Before that, there have been just two publications where numerical multiband exciton dispersion in quantum wells were calculated: in Ref. 17] $\beta=1$ (in the parabolic case $m_{e}=\infty$ ) was taken in order for the form factors (17) to be independent of $Q$, and in Ref. [18] no particular 
choice or handling of $\beta$ is mentioned. In analytic expressions, usually the symmetric (in the parabolic case $m_{e}=m_{h}$ ) value $\beta=1 / 2$ is taken [16].

The effect of the $\beta$ choice becomes clear when one evaluates Eq. (7) for two different values $\beta, \beta^{\prime}=\beta+\delta \beta$ giving $\vec{R}^{\prime}=\vec{R}+(\delta \beta) \vec{\rho}, \vec{k}^{\prime}=\vec{k}-(\delta \beta) \vec{Q}$. Clearly, $\beta$ moves artificially part of the plane wave of the COM motion into the relative part of the exciton (6) or, equivalently, it shifts the relative part of the wavefunction in $\vec{k}$-space. A good choice of $\beta$, as in the parabolic case (8), keeps the relative part of the exciton in real space as smooth as possible or, equivalently, pins the relative part of the wavefunction in $\vec{k}$-space to the origin. This situation is illustrated in Fig. 1 where we plot the envelope of the $H H_{1} C_{1}-1 s$ exciton in the single subband approximation using the symmetric value of $\beta=1 / 2$. This value of $\beta$ is indeed not optimal, as the large shift demonstrates. Diamonds mark where the position of the origin would be for other values of $\beta$.

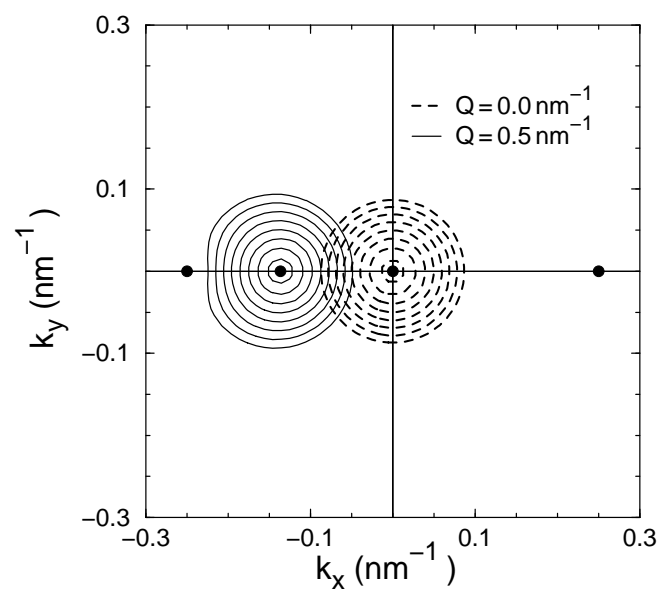

FIG. 1. Logarithmic contour plots of the squared $H H_{1} C_{1}-1 s$ exciton envelope in $\vec{k}$-space for a $5 \mathrm{~nm} \mathrm{GaAs}_{\mathrm{A}} \mathrm{Al}_{0.3} \mathrm{Ga}_{0.7} \mathrm{As}$ QW at $Q_{x}=0.5 \mathrm{~nm}^{-1}$ plotted in a coordinate system with the not optimized value $\beta=1 / 2$. Diamonds mark, from left to right, the origin of the shifted coordinate systems $\left[\right.$ at $\left.(1 / 2-\beta) Q_{x}\right]$ for $\beta=0, \beta_{0}, 1 / 2$, 1 , with $\beta_{0}$ evaluated from Eq. (24). The $Q=0$ envelope is plotted for comparison (dashed).

We introduced in Ref. [19] a quasi-analytical method for determining the optimal choice of the scalar $\beta$, which we briefly summarize here. It is motivated by the fact that in the parabolic case the correct COM transformation decouples the relative motion and COM motion completely. A full decoupling is not possible for non-parabolic dispersions. We looked for a choice of $\beta$ that decouples "as much as possible". To quantify this, we inserted in Eq. (11) the general $\beta$-dependent COM transformation (7), separated the $Q$-dependent terms from the rest

$$
H=H^{(0)}+H^{(1)}(\beta) Q+H^{(2)}(\beta) Q^{2},
$$

and viewed these as a perturbation of the $Q=0$ exciton. Taking into account the inversion symmetry of the Brillouin zone, the kinetic mass of the groundstate exciton $g$ is given in second order perturbation theory by

$$
\frac{\hbar^{2}}{2 M_{g}^{X}}=\left\langle g\left|H^{(2)}(\beta)\right| g\right\rangle+\sum_{a \neq g} \frac{\left|\left\langle a\left|H^{(1)}(\beta)\right| g\right\rangle\right|^{2}}{E_{g}^{X}(0)-E_{a}^{X}(0)} .
$$

However, the exciton mass must not depend on $\beta$. Maximizing the first order contribution in (20) and minimizing this way the strictly positive contribution of the higher states to the groundstate mass leads to the analytical result

$$
\beta=\left\langle g\left|H_{h}^{(2)}\right| g\right\rangle /\left\langle g\left|H_{e}^{(2)}+H_{h}^{(2)}\right| g\right\rangle .
$$

$H_{e, h}^{(2)}$ are simply the material-dependent coefficients of the $\beta^{2} Q^{2}$ terms when inserting (14) into the $k$-space representation of the kinetic energies in Eq. (2) and (3), respectively [19].

The explicit form of Eq. (20) with the contributions from the higher states dropped and $\beta$ from Eq. (21) suggests to define COM-related, effective masses $m_{e, h}^{*}$ for electron and hole

$$
1 / m_{e, h}^{*}=\frac{2}{\hbar^{2}}\left\langle g\left|H_{e, h}^{(2)}\right| g\right\rangle \quad \text { satisfying } \quad M_{g}^{X}=m_{e}^{*}+m_{h}^{*} .
$$


Numerical results show that the masses obtained from (22) tend to be too small. Nevertheless, the obtained values for $\beta$ in [19,31] were quite reasonable because of the much heavier hole mass. If one actually calculates the contributions of the higher exciton states to $M_{g}^{X}$ in (20) (which are dropped in (22)), one finds that the only important correction comes from the coupling to the $L H_{1} C_{1}-1 s$-like state. Taking in Eq. (20) this single correction term into account gives practically the exact curvature of the exciton groundstate dispersion at $Q=0$.

The above procedure is not the best for determining the optimal value of $\beta$ as the importance of the coupling to higher states demonstrates. It was inspired by the solution of the exciton problem in real space. Let us now look at the form of the exciton wavefunction for $Q \neq 0$ in the subband expansion (13). The $Q$-dependence enters the wavefunction: (i) the need to appropriately combine the subband states to get the right $Q$ and (ii) through the need for the envelope to adjust for the anharmonicities in the dispersions. In the perturbation approach described above, we tried to find a COM transformation that keeps for small $Q$ values the entire wavefunction unchanged as much as possible. However, once the one-particle problem is solved, the $Q$-dependence due to the appropriate combination of the subband states (i) is explicitly known. Therefore, a better Ansatz for the wavefunction would be to find a COM transformation that keeps the envelopes as much unchanged as possible; that is Eq. (13) with

$$
\varphi_{n_{e} n_{h}}^{\vec{Q} ; g}(\vec{k})=\varphi_{n_{e} n_{h}}^{0 ; g}(\vec{k}) .
$$

The minimization of the energy with respect to $\beta$ can be done analytically in the limit $Q \rightarrow 0$. We easily obtain the optimized value

$$
\beta_{0}=\frac{\sum_{n_{e} n_{h}} \int d \vec{k}\left|\varphi_{n_{e} n_{h}}^{0 ; g}(\vec{k})\right|^{2}(\hat{Q} \cdot \vec{\nabla})^{2} \mathcal{E}_{n_{h}}(\vec{k})}{\sum_{n_{e} n_{h}} \int d \vec{k}\left|\varphi_{n_{e} n_{h}}^{0 ; g}(\vec{k})\right|^{2}(\hat{Q} \cdot \vec{\nabla})^{2}\left(\mathcal{E}_{n_{e}}(\vec{k})+\mathcal{E}_{n_{h}}(\vec{k})\right)} .
$$

This expression accounts also for the dependence of $\beta_{0}$ on the direction $\hat{Q}$ of the COM momentum in the case of warped valence bands. We took into account the inversion symmetry of the Brillouin zone and assumed that the Coulomb potential (16), (17) can be approximated as a function of the momentum transfer only, $V\left(\vec{k}, \vec{k}^{\prime}\right) \simeq V\left(\vec{k}-\vec{k}^{\prime}\right)$, i.e., we neglected any $Q$-dependence of the (Coulomb) potential energy of the groundstate. This is expected to be a good approximation since the Coulomb energy depends solely on the charge distribution, which should not be affected significantly by the in-plane motion. Indeed, it has been estimated in Ref. 11 that the error introduced by neglecting in Eq. (177) the $\vec{k}$-dependence of the hole envelopes is about $5 \%$. Our assumption should lead to even smaller deviations.

Fig. 1 demonstrates the quality of the expression (24). It shows the groundstate envelope at a rather large value of $Q=0.5 \mathrm{~nm}^{-1}$, even though Eq. (24) was obtained in the limit $Q \rightarrow 0$. It is particularly impressive that the respective origin for the optimized choice $\beta_{0}$ from Eq. (24) lies even a bit to the left of the maximum of the envelope. This accounts for the $p$-component that deforms the originally radially symmetric $Q=0$ envelope; using an angular momentum decomposition of the envelope a minimum number of components would be needed. The slight deformation (these are logarithmic plots) for large values of $Q$ is due to the anharmonicity of the one-particle dispersions.

The importance of a suitable choice of the COM transformation for the numerical convergence is illustrated in Fig. 2 for the dispersion of the $H H_{1} C_{1}-1 s$ exciton of a $5 \mathrm{~nm}$ QW. This has been calculated in $\vec{k}$-space for various values of $\beta$ with the same basis $\left(H H_{1} C_{1}, L H_{1} C_{1}, \ell=0, \pm 1, \pm 2\right)$. The further the used $\beta$ lies from the optimal value $\beta_{0}\left(\beta_{0}=0.23\right.$ in this case) the worse the results are. We did also calculations where for each $Q$ value an optimal value of $\beta$ was obtained by numerical variation. We observed deviations from $\beta_{0}$ less than $1 \%$ near $Q=0$ and not larger than $10 \%$ at $Q=0.5 \mathrm{~nm}^{-1}$ even for the widest well $\left(20 \mathrm{~nm}\right.$ ). At large $Q$ the $\beta=0$ (in the parabolic case $m_{h}=\infty$ ) curve gives slightly better results than $\beta_{0}$ since the $H H_{1}$ subband dispersion gets more flat after the avoided crossing with the $L H_{1}$ subband, but it gives considerably worse results at small $Q$. 


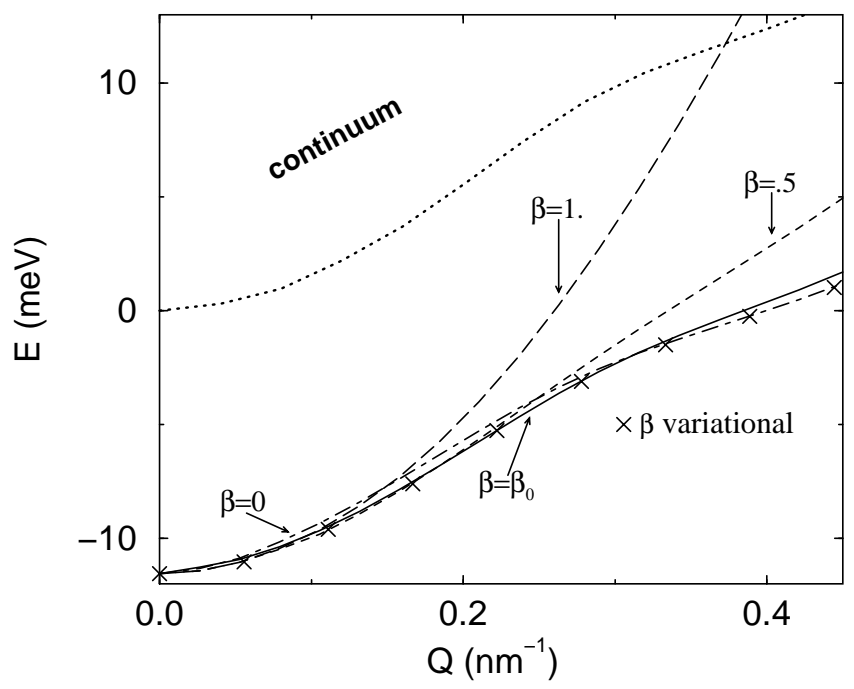

FIG. 2. Dispersion of the groundstate exciton of a $5 \mathrm{~nm}$ wide QW calculated with the same basis but for various values of $\beta$. The exciton continuum edge (dotted), Eq. (28), is given for comparison.

\section{A SIMPLE ANALYTICAL FORMULA FOR THE AVERAGE EXCITON KINETIC MASS}

The analytical variation that led to Eq. (24) gives the groundstate energy up to terms quadratic in $Q$. The corresponding groundstate kinetic mass $M_{g}^{X}$ has again the form

$$
M_{g}^{X}=m_{e}^{X}+m_{h}^{X}
$$

with the COM-related effective masses for electron and hole defined as

$$
1 / m_{e, h}^{X}=\frac{1}{\hbar^{2}} \sum_{n_{e} n_{h}} \int d \vec{k}\left|\varphi_{n_{e} n_{h}}^{0 ; g}(\vec{k})\right|^{2}(\hat{Q} \cdot \vec{\nabla})^{2} \mathcal{E}_{e, h}(\vec{k})
$$

With these masses, the expression for $\beta_{0}$, Eq. (24), has the same form as in the parabolic case (8). Eq. (26) gives the correct results for the free particle case.

We claim that this simple result will be of considerable practical importance. Eq. (26) is physically appealing: it leads to a weighted average of the subband dispersions. Further, it is relatively simple to calculate: It requires only the approximate knowledge of the $Q=0$ exciton envelope and of the involved subband dispersions. The numerical calculation of subband dispersions is nowadays an easy task (provided the $\vec{k} \cdot \vec{p}$ parameters are known). Moreover, especially for narrow QW, the envelope of the $H_{1} C_{1}$ component of the groundstate exciton is to a very good approximation similar in shape to the groundstate of the 2D exciton,

$$
\varphi_{1 s}^{2 D}(\vec{\rho})=\sqrt{\frac{2}{\pi a_{B}^{2}}} e^{-a_{B} \rho}, \quad \varphi_{1 s}^{2 D}(\vec{k})=\sqrt{\frac{2 a_{B}^{2}}{\pi}}\left(1+\left(a_{B} k\right)^{2}\right)^{-3 / 2} .
$$

The $L H_{1} C_{1}$ component is quite small, e.g., $5 \%$ for the $20 \mathrm{~nm} \mathrm{QW}$, and can be safely neglected in this context. Therefore, only a good estimate for the effective Bohr radius $a_{B}$ is needed to evaluate (26).

\section{RESULTS}

We have calculated exciton dispersions both in real and momentum space for GaAs $/ \mathrm{Al}_{0.3} \mathrm{Ga}_{0.7} \mathrm{As}\langle 001\rangle \mathrm{QW}$ of various widths. The coupling of heavy and light holes was fully incorporated. The values of the material parameters $\gamma_{1}, \gamma_{2}, \gamma_{3}, m_{e}$ were taken by linear interpolation from the GaAs and AlAs values; the offset ratio was $V_{v} / V_{c}=0.68 / 0.32$ and the band gap in meV was taken as $E_{g}(x)=1519+1040 x+470 x^{2}, x$ being the Al content [32]. For the dielectric constant, we adopted $\epsilon=12$ for both well and barrier material. 


\section{A. Subband expansion}

The nomenclature is as follows: the exciton in the subband expansion has various $n_{h} n_{e}$ subband components with the corresponding envelopes $\varphi_{n_{e}}^{\vec{Q} ; a} n_{h}(\vec{k})$, Eq. (13). These envelopes have in the axial approximation at $Q=0$ a definite angular momentum $\ell$ and will be denoted by $1 s, 2 s, 2 p_{ \pm}, 3 d_{ \pm}$and so on. Each exciton state at finite $Q$ will be named according to the main subband component of the corresponding state at $Q=0$. That is, speaking of the $H H_{1} C_{1}-1 s$ exciton means that at $Q=0$ its main subband component is the $H H_{1} C_{1}$ one with an $1 s$ envelope. Similar to the single-particle hole subband states, which can change their heavy- or light-hole character away from the $\Gamma$-point, the envelope of the main subband component or even the main subband component itself can change with increasing $Q$. To denote the main subband component of a state at a given value of $\vec{Q}$, we will speak of the character of the state at this $\vec{Q}$. For example, the $H H_{1} C_{1}-2 p_{+}$exciton has a $H H_{1} C_{1}-2 p_{+}$character at $Q=0$ and a $L H_{1} C_{1}-1 s$ character $Q \gg 0$.

The exciton dispersions in $k$-space are calculated as follows: For each QW, we first calculate the exciton spectrum at $Q=0$. Subsequently, a $2 \mathrm{D} 1 s$-exciton groundstate function (27) is fitted to the $H H_{1} C_{1}$ envelope. For the wider QW, also a two-dimensional $3 d$-exciton function is fitted to the $L H_{1} C_{1}$ envelope. This fit is used, instead of the numerical envelope, to evaluate the optimized COM transformation (24) because it allows to take advantage of the analytically known derivatives of the fit function. The so calculated value of $\beta_{0}$ is used for the $Q \neq 0$ calculations.

In Fig. 3, we display the envelopes of the components of the groundstate exciton in the subband expansion (13) for a $15 \mathrm{~nm}$ wide QW at $Q=0$. The coupling of the higher subbands is rather small, less than $3 \%$ for the $L H_{1} C_{1}$ component and even less for the others. The parity and spin selection rules for the Coulomb coupling of the subbands at the $\Gamma$-point in symmetric QW are obeyed, e.g., the admixture of the $L H_{1} C_{1}$ state vanishes at the $\Gamma$-point since the Coulomb potential is spin-diagonal and the $H H_{1}$ and $L H_{1}$ subband states are pure heavy- and light-hole states, respectively. The $H H_{1} C_{1}$ envelope is very well approximated by a $2 \mathrm{D} 1 s$-exciton function. Deviations are mainly located at the vicinity of the $H H_{1}-L H_{1}$ avoided crossing of the hole-subband dispersions (here, $k_{a c} \simeq 0.13 \mathrm{~nm}{ }^{-1}$ ). The total in-plane probability distribution follows the form of Eq. (27) even better than the $H_{1} H_{1}$ envelope alone; the coupling to the higher subbands allows the exciton to relax further. This, again, supports the notion that the subband mixing has little influence on the charge distribution.

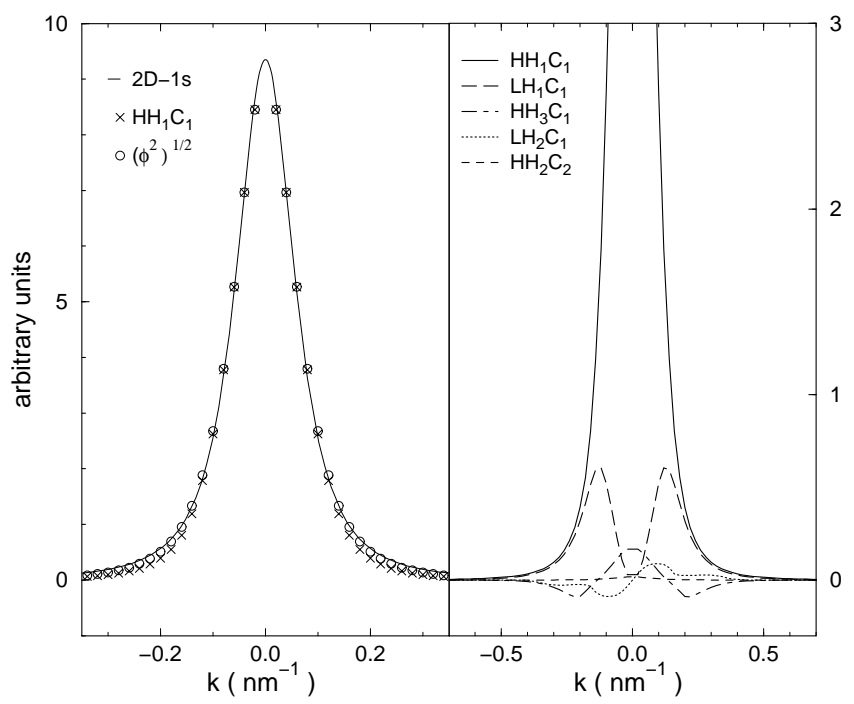

FIG. 3. On the right: Envelopes of the subband components of the groundstate exciton calculated in $k$-space for a $15 \mathrm{~nm}$ wide QW at $Q=0$. On the left: $H H_{1} C_{1}$ envelope $(\times)$, fitted $2 \mathrm{D} 1 s$-exciton wavefunction (line), and square root of the total probability distribution $|\phi(k)|^{2}=\sum_{n_{e} n_{h}} \varphi_{n_{e} n_{h}}^{0 ; g}(k)^{2}$ (circles).

The calculations in $k$-space presented in the following take into account only the two lowest hole subbands $\left(n_{h} n_{e}=\right.$ $\left.H H_{1} C_{1}, L H_{1} C_{1}\right)$. Inclusion of higher subbands does not enhance the binding energy of the groundstate exciton considerably. For the angular decomposition of the envelope components only the $s, p_{ \pm}, d_{+}(\ell=0, \pm 1,2)$ components for the $H H_{1} C_{1}$ and the $s, p_{ \pm}, d_{-}(\ell=0, \pm 1,-2)$ components for the $L H_{1} C_{1}$ were considered. Due to the optimized choice of the COM coordinate system, Eq. (24), these few angular momentum components are sufficient to describe the $H_{1} C_{1}-1 s$ and $L H_{1} C_{1}-1 s$ dispersions excellently over the whole range of COM momentum values considered, 
$Q \leq 0.5 \mathrm{~nm}^{-1}$ : The $p$ components account mainly for the deformation of the envelope, and the $d$ components take care of the Coulomb coupling to higher states.

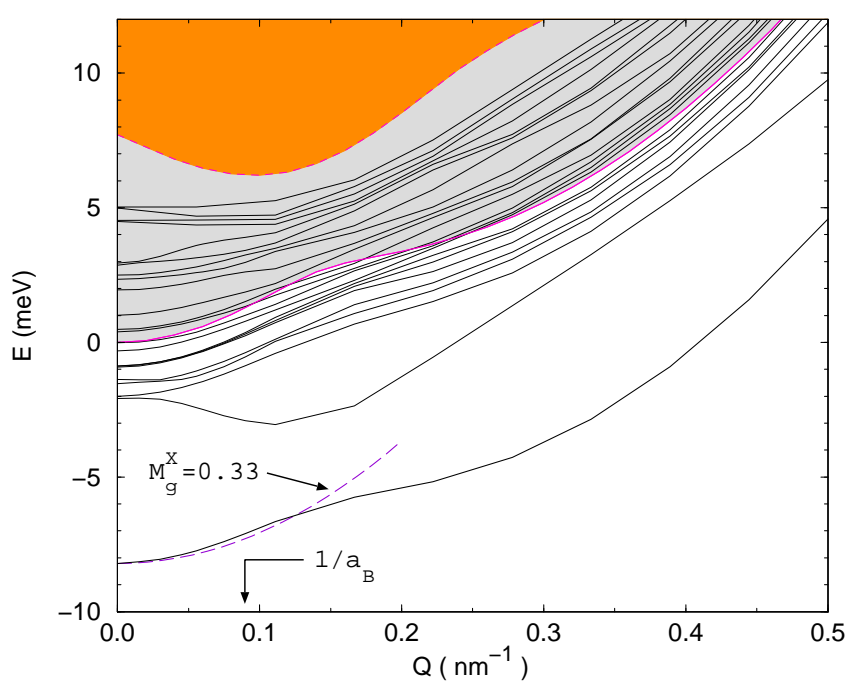

FIG. 4. Exciton dispersions, groundstate (thick) and some excited bound and continuum states (thin), calculated in $k$-space for a $15 \mathrm{~nm}$ wide QW. The light (dark) gray shaded area mark the $H H_{1} C_{1}\left(L H_{1} C_{1}\right)$ exciton continuum. A parabola for the average groundstate exciton mass $M_{g}^{X}$ after Eqs. $(25,26)$ is plotted (dashed). An arrow marks the position of $1 / a_{B}\left(a_{B}\right.$ the Bohr radius).

In Fig. 1. we show the dispersion calculated in $k$-space of the first bound exciton states as well as some of the continuum states in a $15 \mathrm{~nm}$ wide QW. Zero of energy is the onset of the $H H_{1} C_{1}$ continuum at $Q=0$. The Bohr radius $a_{B}$ is the one obtained from the same fit of a $2 \mathrm{D} 1 s$-exciton to the envelope of the $H H_{1} C_{1}$ component at $Q=0$ which was used to calculate the optimized $\beta_{0}$. The groundstate is $H H_{1} C_{1}-1 s$. The next state is $H H_{1} C_{1}-2 p_{+}$which turns into $L H_{1} C_{1}-1 s$ character at the avoided crossing. The $L H_{1} C_{1}-1 s$ exciton is at $Q=0$ the fourth excited state and shows a substantial mixing with the $H H_{1} C_{1}-3 d_{+}$exciton. The $H H_{1} C_{1}-2 p_{+}$exciton has at $Q=0$ a slightly lower energy than the $H H_{1} C_{1}-2 p_{-}$(third state at $Q=0$ ) because it couples to the $L H_{1} C_{1}-1 s$ outside of the $\Gamma$-point.

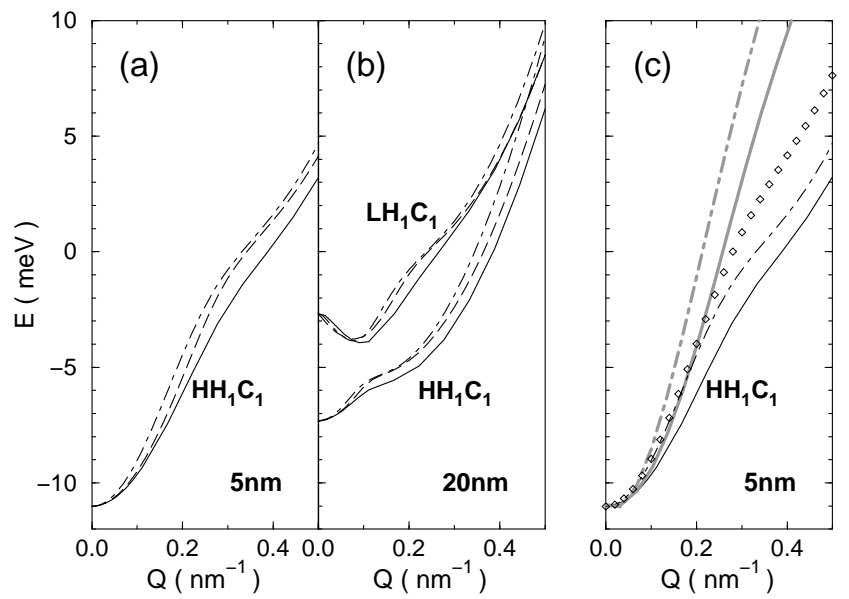

FIG. 5. (a), (b): Comparison of the dispersion of $H H_{1} C_{1}-1 s$ and $L H_{1} C_{1}-1 s(20 \mathrm{~nm}$ QW only) excitons (thick) with the appropriately shifted respective exciton continuum edge (dashed) and the underlying hole-subband dispersion (dot-dashed) for two GaAs/Al $\mathrm{Al}_{0.3} \mathrm{Ga}_{0.7} \mathrm{As}$ QW. (c): Comparison of our results (black) with those of Ref. [18] (gray) for the $5 \mathrm{~nm}$ QW (shifted to match at $Q=0$ ). The exact $H H_{1}$-subband dispersion for the parameters of 18 ] is plotted, too (diamonds).

As was reported in earlier work [17,18], the exciton COM dispersions are highly non-parabolic, much like the hole subband dispersions. This is not surprising, since the dispersion of the conduction subband is parabolic and the hole mass is much larger than the electron mass. Furthermore, it has been claimed in Ref. 18] that the exciton dispersion 
follows, in a good approximation, the hole subband dispersion. Although this is certainly true in the present case due to the parabolic electron dispersion and the small electron to hole mass ratio, the exciton dispersion is in principle a two-particle quantity. In fact, the dispersion of the groundstate exciton follows even more closely, in the studied cases within $1 \mathrm{meV}$, the electron-hole-pair continuum edge $\mathcal{E}_{n_{e} n_{h}}(\vec{Q})$. The latter is defined by

$$
\mathcal{E}_{n_{e} n_{h}}(\vec{Q})=\min _{\vec{k}_{e}+\vec{k}_{h}=\vec{Q}}\left\{\mathcal{E}_{n_{e}}\left(\vec{k}_{e}\right)+\mathcal{E}_{n_{h}}\left(\vec{k}_{h}\right)\right\}
$$

and represents the minimal kinetic energy of a free electron-hole pair for a given subband combination $n_{e} n_{h}$ and a given $\vec{Q}$. In the independent-subband approximation, this coincides with the exciton continuum edge. Fig. $5(\mathrm{a}, \mathrm{b})$ directly compare for a narrow and a wide QW the exciton groundstate dispersion and the appropriately shifted exciton continuum edge. Also shown is the respective hole dispersion. The latter lies always above the shifted exciton continuum edge since Eq. (28) implies $\mathcal{E}_{n_{e} n_{h}}(\vec{Q}) \leq \mathcal{E}_{n_{e}}(0)+\mathcal{E}_{n_{h}}(\vec{Q})$.

The exciton groundstate dispersion is found to lie systematically below the shifted exciton continuum edge, i.e., the groundstate exciton binding energy becomes larger away from $Q=0$. This can be understood based on the fact that for $Q \neq 0$ the groundstate exciton, Eqs. $(13,14)$, is built from hole subband states around $\left(1-\beta_{0}\right) Q$. Due to the flatter subband dispersion around this point, the hole mass gets larger and the wavefunction can better adjust to the potential. The $L H_{1} C_{1}-1 s$ exciton in the $20 \mathrm{~nm}$ wide QW, Fig. 5 $\mathrm{b}$, is well separated from the spectrum of the $H H_{1} C_{1}$ exciton. We observe that at small $Q$ the $L H_{1} C_{1}-1 s$ dispersion lies above the respective shifted exciton continuum edge. Indeed, the $L H_{1}$ subband shows a negative mass at the $\Gamma$-point which becomes positive near the avoided crossing. Hence, the respective exciton has to pay with extra kinetic energy in order to achieve a small COM momentum, and its binding energy is decreased. For larger $Q$ values it gains again some binding energy.

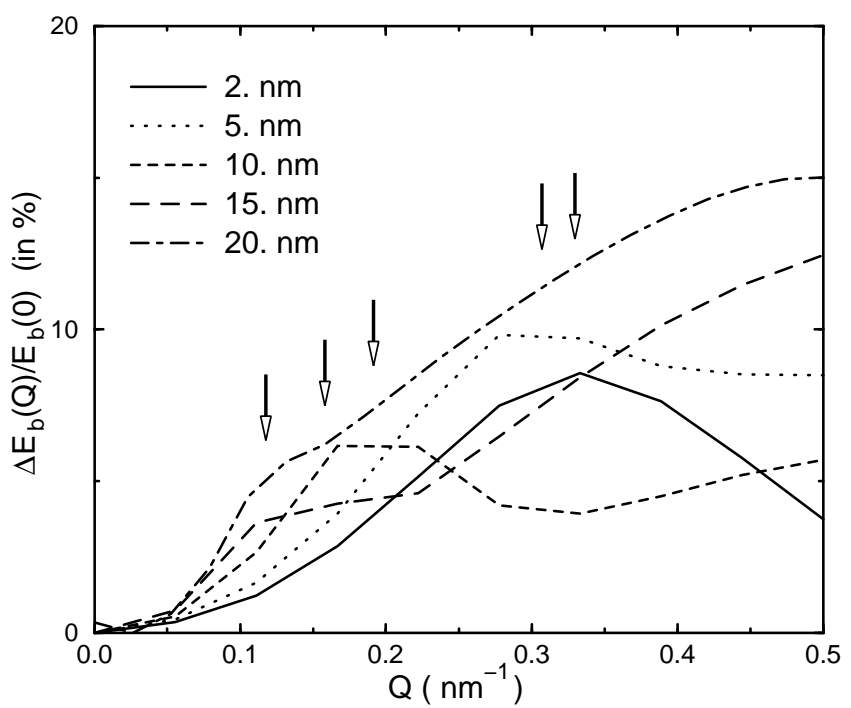

FIG. 6. Enhancement of the exciton binding energy $E_{b}(Q)$ with increasing center of mass momentum $Q$ for $\mathrm{GaAs} / \mathrm{Al}_{0.3} \mathrm{Ga}_{0.7} \mathrm{As} \mathrm{QW}$ of various widths. The arrows mark the position of the respective $H H_{1}-L H_{1}$ avoided crossing, from the right to the left for growing QW width.

The enhancement of the groundstate binding energy for $Q \neq 0$ is particularly large when the exciton is built from hole subband states around the avoided crossings $\left(1-\beta_{0}\right) Q=k_{\text {a.c. }}$. This is demonstrated in Fig. 6 for QW of various widths. Peaks are seen at the respective location of the $H H_{1}-L H_{1}$ avoided crossing, marked by arrows. The enhancement of the groundstate binding energy with $Q$ is less than $15 \%$ and is generally larger for wider $\mathrm{QW}$. 


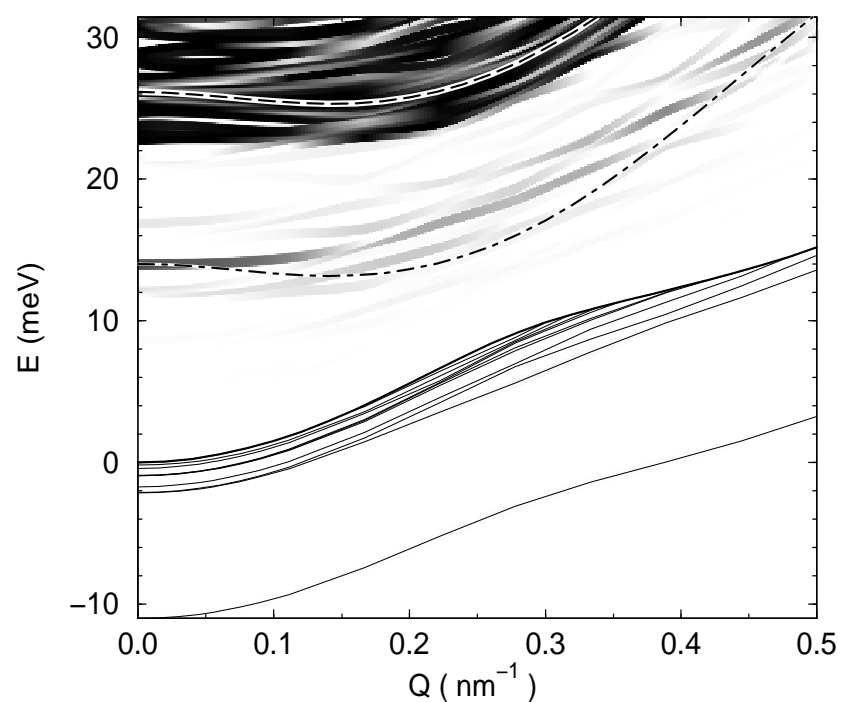

FIG. 7. Exciton dispersion for $5 \mathrm{~nm} \mathrm{GaAs} / \mathrm{Al}_{0.3} \mathrm{Ga}_{0.7} \mathrm{As}$ QW. The $H H_{1} C_{1}$ (thick) and $L H_{1} C_{1}$ (dashed) exciton continuum edges are also shown. The background shading indicates how strong is the mixture of the $L H_{1} C_{1}$ exciton states to the numerically calculated eigenstates (black corresponds to 100\%). For comparison, also a $L H_{1} C_{1}$ (dot-dashed) exciton continuum edge shifted to the energy of the $L H_{1} C_{1}-1 s$ state at $Q=0$ has been drawn.

In Fig. 7, we plot the exciton dispersion of a $5 \mathrm{~nm}$ QW. The exciton dispersions, like the hole subband dispersions, are less anharmonic in the much narrower QW than for the QW of Fig. A because of the larger energy separation of the hole subbands. The avoided crossing takes place at rather large $k\left(k=0.31 \mathrm{~nm}^{-1}\right)$. The shading indicates the percentage of the contribution of the $L H_{1} C_{1}$ subband states to the norm of the numerical eigenvectors. The very light shading of the bound exciton states confirms the small admixture of the $L H_{1} C_{1}$ states in the groundstate. The shading is darker in the vicinity of the avoided crossing. Small-scale intensity variations are numerical artefacts due to the finite $k$-space mesh. In the exciton continuum only the shading is shown. The $L H_{1} C_{1}-1 s$ resonance is clearly seen starting at approximately $18 \mathrm{meV}$. The resonance is sharper at the beginning and becomes more diffuse at the avoided crossing. It lies slightly above the respective exciton continuum edge.

\section{B. Real-space calculations}

For effectively two-dimensional structures with translational symmetry like the considered symmetric QW in axial approximation and, maybe, for some highly idealized quantum wire structures, the real-space method presented in section IIB can not compete with the one in $k$-space. However, for realistic one-dimensional structures, like Vgroove and T-shaped quantum wires, this may be the only feasible approach for calculating the exciton groundstate dispersion. This is due to the high number of confining dimensions for the exciton (in quantum wires four, two for each particle): an expansion in a problem-adapted basis like the product basis of the one-particle eigenstates, for which one expects reasonable convergence, leads to four-dimensional integrals for the Coulomb interaction. An expansion in a basis where the Coulomb potential is simple will probably show a very slow convergence with basis size.

The calculations reported here are mainly to be viewed as tests of the applicability of our real-space approach and its generalization to finite-elements discretization. They are primarily compared with results obtained with the more established $k$-space methods. We will therefore discuss the results of the real-space calculations focusing on the convergence properties of the method. Further, wavefunction features are better visualized in real space, in particular, the electron-hole correlation in the growth direction.

For the real-space calculations at finite $Q$, we used the optimized $\beta_{0}$ obtained in the respective $k$-space calculations. We could have used equally well some other procedure to find the effective Bohr radius $a_{B}$, e.g., a variational one, or we could also have fitted a 2D 1s-exciton function to the in-plane probability distribution of the previously calculated exciton groundstate at $Q=0$.

The Coulomb potential was discretized as described in the Appendix. The 3D $1 s$-exciton in the four dimensional space $\left(\vec{\rho}, z_{e}, z_{h}\right)$ with $m_{e}^{r e f}=0.0665 m_{0}, m_{h}^{r e f}=0.24 m_{0}$ was used as reference groundstate. The value for the reference hole mass was taken from Fig. 11, discussed below, as an average value for the range of QW widths considered. This gives a reasonable reference Bohr radius of $a_{B}^{r e f}=12.2 \mathrm{~nm}$; it is nearly the correct value for the in-plane motion or 
somewhat larger. In the confinement direction the size of the reference wavefunction is larger than the actual one (the exciton is quenched in this direction), too. As discussed in the Appendix, a reference Bohr radius as large as or somewhat larger than the actual one gives good convergence. We did test calculations with $a_{B}^{\text {ref }}$ doubled and with $a_{B}^{r e f}$ halved and found a qualitatively similar behavior as in Fig. 12 in the Appendix. The integration region was $120 \mathrm{~nm}$ wide in the $\vec{\rho}$ directions and $30 \mathrm{~nm}(60 \mathrm{~nm})$ wide in the $z$ directions for the $5 \mathrm{~nm}(20 \mathrm{~nm})$ QW with a gridpoints distance of about $a_{B} / 6$ in the in-plane directions.

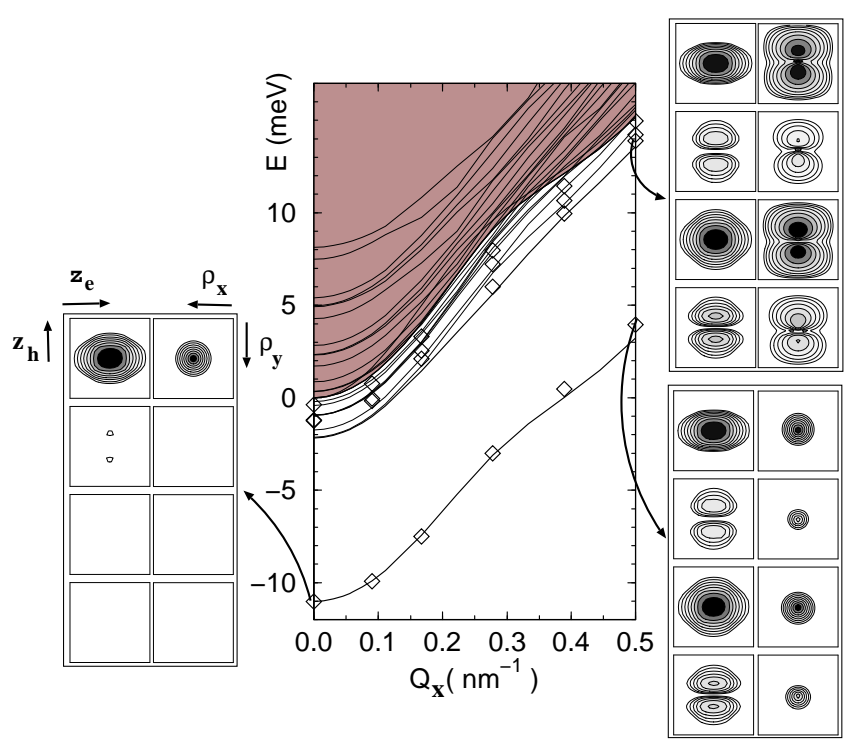

FIG. 8. Middle panel: Exciton dispersion for a $5 \mathrm{~nm}$ wide QW. $k$-space results for the groundstate and some excited bound and continuum states (lines) and the exciton continuum (shaded) are presented, as well as some real-space results (diamonds). Side panels with logarithmic contour plots of the exciton probability distribution for each spin-component (from top to bottom $m_{J}=+\frac{3}{2},+\frac{1}{2},-\frac{1}{2},-\frac{3}{2}$ ) are shown for characteristic exciton states (thick lines) at $Q=0$ (left) and $Q_{x}=0.5 \mathrm{~nm}^{-1}$ (right panels). The displayed area is in each direction $10 \mathrm{~nm}$ wide for the $z_{e} z_{h}$ and about $10 a_{B}$ wide for the $\rho_{x} \rho_{y}$ plots, respectively.

The panel in the middle of Fig. 8 shows dispersions of the lowest exciton states (diamonds) from the real-space calculation for a $5 \mathrm{~nm}$ wide QW. The exciton dispersions calculated in momentum space are plotted as full curves for comparison. The groundstate binding energy is not yet fully converged: all real-space results were shifted approximately $1 \mathrm{meV}$ to lower energies to match the groundstate energies at $Q=0$ for both methods. Numerical tests show that the gridpoint density in the growth direction $(\approx 0.6$ points $/ \mathrm{nm})$ is more critical than in the in-plane direction $(\approx 0.6$ points $/ \mathrm{nm})$. The exciton continuum edge lies also $0.5 \mathrm{meV}$ too high, and the stronger confinement due to the Coulomb interaction lets us expect for the exciton a larger deviation. Nevertheless, the groundstate relative dispersion, $\mathcal{E}_{g}^{X}(Q)-\mathcal{E}_{g}^{X}(0)$, is converged and reproduces the $k$-space results very well. We note that in the case of parabolic one-particle dispersions, this is an exact property of any numerical exciton dispersions. The dispersions of the excited states are not reproduced that well. This is mainly due to their larger spatial extension and smaller energy separation from each other compared to the groundstate.

The panels on the left and on the right in Fig. 8 show logarithmic contour plots of the exciton probability distribution for some characteristic states. The probability distribution is either integrated over $z_{e}, z_{h}$ and displayed in the $\vec{\rho}$ plane, or integrated over $\vec{\rho}$ and displayed in the $z_{e} z_{h}$ plane for each spin component separately. The numerically obtained wavefunctions are a linear combination of the two degenerate solutions with opposite parity, Eq. (10). They are disentangled according to parity $P$, and only $P=-1$ states are displayed.

The left panel displaying the groundstate exciton at $Q=0$ illustrates its $H H_{1} C_{1}-1 s$ character: the main spin component is $m_{J}=+3 / 2$ and has no nodes. The bulk of the exciton is confined in the QW but there is substantial penetration into the barrier, being stronger for the lighter electron (note the logarithmic plot). At $Q=0.5 \mathrm{~nm}^{-1}$ (right panels), the groundstate exciton has still $H_{1} C_{1}-1 s$ character, as is seen in the $k$-space calculations, Fig. 3. This does not contradict the strong mixture of heavy- and light-hole bulk states seen in the lower right panel of Fig. 8. Indeed, the exciton is built from hole subband states near $\left(1-\beta_{0}\right) Q$. This point lies past the $H H_{1}-L H_{1}$ avoided crossing. Hence, the $\mathrm{HH}_{1}$ subband states near this point are a strong mixture of heavy- and light-hole bulk bandedge states. The stronger penetration into the barrier of the light-hole component is again related to its smaller mass.

An interesting feature is the larger confinement of the $m_{J}=+\frac{3}{2}$ component at $Q=0.5 \mathrm{~nm}^{-1}$ compared to $Q=0$. 
This is a consequence of the enhanced exciton binding energy. Altogether, the plots demonstrate that the total charge distribution in not altered much with increasing COM momentum; the somewhat stronger confinement of the heavy hole is at least partly canceled by the larger penetration into the barrier of the light hole. Although here not clearly resolved, the in-plane plots at $Q=0.5 \mathrm{~nm}^{-1}$ show the slight deformation of the originally radially symmetric wavefunction that was seen in Fig. 11. Again these deformations partly cancel each other in the sum over the spin components, and the in-plane charge distribution remains mainly symmetric. This is more clearly seen for the first excited state at $Q=0.5 \mathrm{~nm}^{-1}$ which has $H H_{1} C_{1}-2 p_{y}$ character (upper right panel in Fig. 8). It is the $H H_{1} C_{1}-2 p_{+}$ exciton, which is, again, slightly lower in energy than the $H H_{1} C_{1}-2 p_{-}$exciton at $Q=0$. At large $Q$ the character of the $H H_{1} C_{1}$ envelope changes from $2 p_{ \pm}$to $2 p_{y, x}$.

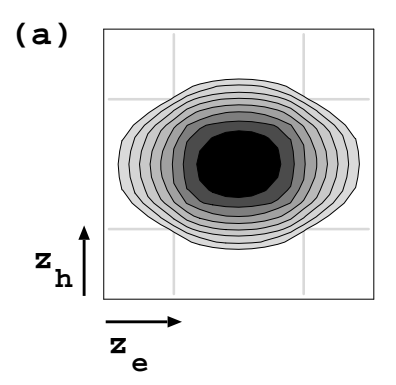

\section{(b)}

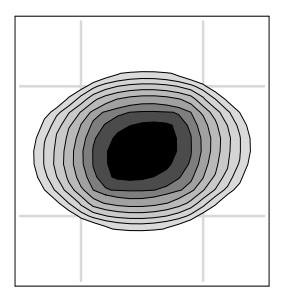

FIG. 9. Groundstate exciton probability density in the $z_{e} z_{h}$-plane for the main spin-component $\left(m_{J}=+\frac{3}{2}\right)$ at $Q=0$ for a $5 \mathrm{~nm}$ wide QW, (a) integrated over the in-plane relative coordinate $\vec{\rho}$ (same as lower left corner of Fig. 8) and (b) at $\vec{\rho}=0$. The background lines mark the position of the QW interfaces.

One does not expect strong electron-hole correlation in the growth direction for the $5 \mathrm{~nm} \mathrm{QW}$, which is considerably narrower than the exciton Bohr radius. Here, the confining potentials are on average much stronger than the Coulomb potential and the wavefunction can not relax in this direction. Indeed, in Fig. 9(a) the probability density integrated over $\vec{\rho}$ does not show much correlation: the contour lines are not elongated along the diagonal, $z_{e}=z_{h}$. However, some correlation exists as the plot of the cut at $\rho=0$ in Fig. 9(b) demonstrates. This weak correlation is not included in our $k$-space calculations where only one conduction band was used. Its envelope does not depend on $k$ and consequently the $z_{e}$ coordinate can be separated, Eq. (13).

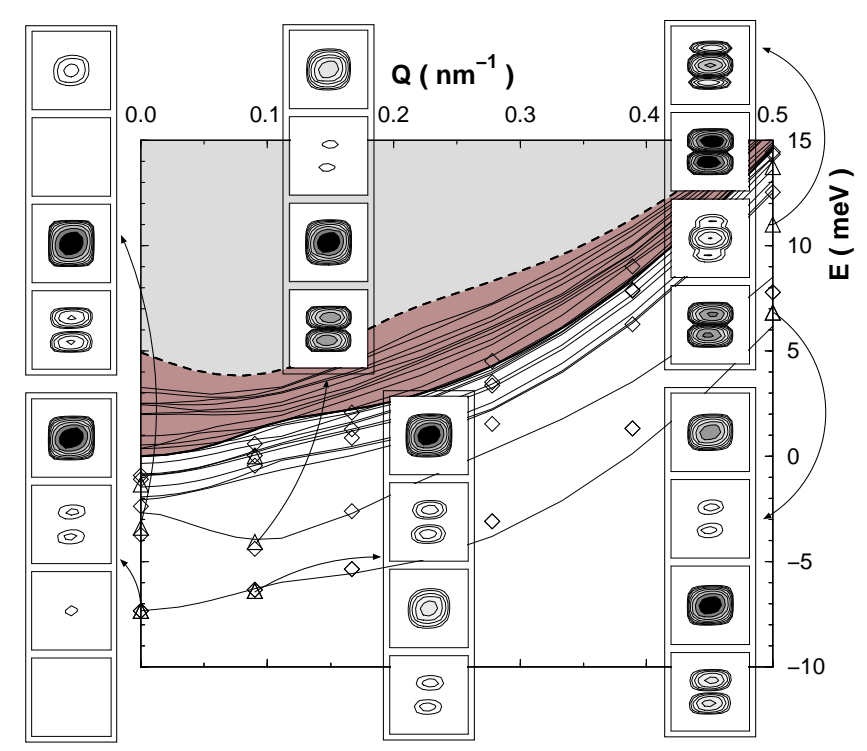

FIG. 10. Logarithmic contour plots of the exciton probability distribution in the $z_{e} z_{h}$-plane for each spin-component (in each panel from top to bottom $m_{J}=+\frac{3}{2},+\frac{1}{2},-\frac{1}{2},-\frac{3}{2}$ ) for the $H H_{1} C_{1}-1 s$ and the $L H_{1} C_{1}-1 s$ excitons at some characteristic $Q$-values for a $20 \mathrm{~nm} \mathrm{QW}$. The displayed region is in each direction twice the QW width. The triangles (diamonds) show results of the real-space calculations for a dense (less dense) mesh. Lines and shading of the background panel are the $k$-space results of Fig. 4. We do not show the $\mathrm{HH}_{2} \mathrm{C}_{1}$ continuum that lies partly in the displayed region. 
In Fig. 10, results are displayed for a $20 \mathrm{~nm}$ wide QW. Two set of points are shown for two different mesh sizes. The energies of the groundstate exciton are almost converged for the more dense mesh; the deviation from the results of the $k$-space calculations is only $0.2 \mathrm{meV}$ at $Q=0$.

The small, but noticeable $m_{J}=+1 / 2$ component of the groundstate exciton $H H_{1} C_{1}-1 s$ exciton in the lower left panel corresponds to the substantial admixing of the $L H_{1} C_{1}-d_{-}$exciton seen already for the $15 \mathrm{~nm} \mathrm{QW}$ in Fig. 3. The admixing is larger for wider QW due to the smaller energy separation of the respective subbands. At $Q=0.5 \mathrm{~nm}^{-1}$, it has mainly bulk light-hole character $\left(m_{J}=-1 / 2\right)$. This, again, does not contradict the $H H_{1} C_{1}-1 s$ character since the $H H_{1}$ subband has approximately $60 \%$ light-hole character beyond the avoided crossing with the $L H_{1}$ subband. The first excited state at $Q=0.5 \mathrm{~nm}^{-1}\left(L H_{1} C_{1}-1 s\right.$ exciton) has $L H_{1} C_{1}-1 s$ character even though an additional node is seen in the $z_{e} z_{h}$ probability distribution of the main spin component $\left(m_{J}=+1 / 2\right)$. The envelopes of the single-particle subband states at large enough in-plane momentum show more nodes than at the $\Gamma$-point due to the coupling of in-plane and growth directions in the Luttinger Hamiltonian. This is one of the reasons why the expansion Eqs. (13,14) gives very good results with just two subbands, while an expansions in the subband states at the $\Gamma$-point 18] needs more subbands for the same accuracy.

The probability distribution plots illustrate the almost vanishing penetration into the barriers for the wide QW, in contrast to the narrower QW of Fig. 8. All panels show a clear orientation of the contour lines towards the diagonal, $z_{e}=z_{h}$. This demonstrates the considerable electron-hole correlation in the growth direction for QW wider than one Bohr radius. Still, the stronger correlation has little impact on the energies. Recall that in perturbation theory the first order correction to the wavefunction gives only a second order correction to the energy. This justifies the usual factoring out of the dependence on the growth direction for the much lighter electron.

This, however, is not the case for the much heavier hole. It is a well known fact, that the Coulomb coupling of the hole subbands is considerable. Indeed, for the $20 \mathrm{~nm}$ wide QW, neglecting the Coulomb coupling of the $H_{1} C_{1}$ - to the $L H_{1} C_{1}$-excitons leads to an error in the groundstate binding energy larger than $10 \%$. That is, the correlation of in-plane and confinement directions for the hole is substantial.

For the $20 \mathrm{~nm}$ QW, a single calculation at $Q=0.5 \mathrm{~nm}^{-1}$ was done with $\beta=1$ in order to check the relevance of this parameter for the numerical accuracy in the real-space calculations, too. Indeed, the respective groundstate energy lies very far ( $43 \mathrm{meV}$ !) above the correct value.

\section{Average exciton groundstate mass}

In the process of determining the optimal choice of the COM coordinate system, we derived in section [II] an expression for the kinetic mass of the groundstate exciton, Eqs. (25,26). Two assumptions were essential: First, the Coulomb potential, i.e., the form factors, are a function of the in-plane momentum transfer, and, second, the Ansatz (23) is valid. The numerical results of the previous sections support these assumptions.

Together with the exciton dispersions in Fig. A, we displayed for the groundstate exciton a parabola with the exciton mass $M_{g}^{X}$ of section $[\mathrm{III}$. This mass is obviously not the one determined by the curvature of the exciton groundstate dispersions at $Q=0$. It is rather an average of the curvature of the groundstate dispersion in a region of size $1 / a_{B}$. Indeed, this is implied by Eqs. (25,26) and the observation made in section $\mathrm{VA}$ that the groundstate dispersion follows closely the respective exciton continuum edge.

This exciton COM momentum region is the one important for exciton localization due to well width fluctuations, interface roughness or alloy fluctuations in QW [1]. Indeed, the exciton averages over smaller scale fluctuations due to its finite size and feels an effective disorder potential that is spatially correlated over the Bohr radius $a_{B}$. 


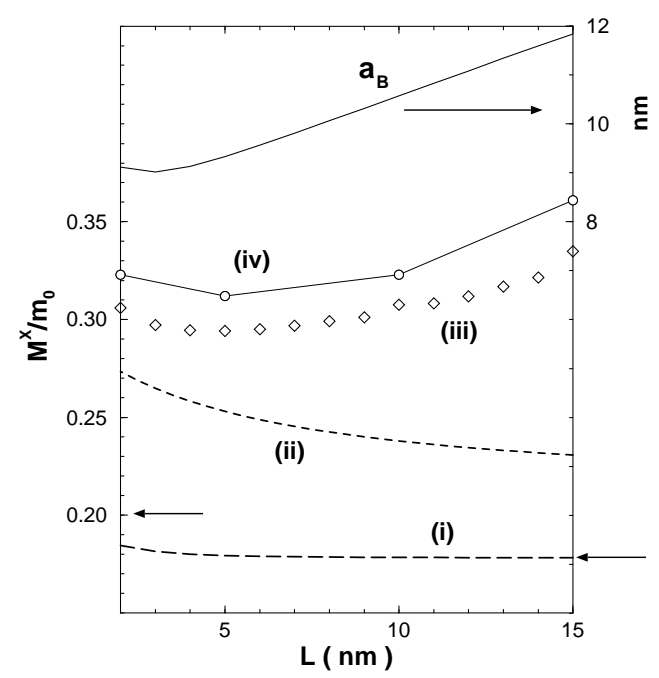

FIG. 11. Exciton average kinetic mass in various approximations and Bohr radius $a_{B}$ versus well width $L$ for $\mathrm{GaAs} / \mathrm{Al}_{0.3} \mathrm{Ga}_{0.7} \mathrm{As} \mathrm{QW}$. The figure is discussed in section $\mathrm{VO}$.

The dependence of various expressions for the groundstate exciton mass on the QW width $L$ is displayed in Fig. 11. The conduction band mass was taken material-independent $m_{e}^{b, w}=0.0665$, the shown $L$-dependence comes solely from the valence band. Displayed are the masses obtained by: (i) describing the hole in the diagonal Luttinger approximation $1 / m_{h}=P_{w}\left(\gamma_{1}^{w}+\gamma_{2}^{w}\right)+P_{b}\left(\gamma_{1}^{b}+\gamma_{2}^{b}\right)$, where $P_{w, b}$ denote the probability that the hole is in the well and barrier material respectively (long dashed), (ii) taking as hole mass the subband curvature at the $\Gamma$-point that is known analytically [33] (dashed), (iii) using our semi-analytical expression (25,26) for the mass with a 2D 1s-exciton function fitted to the envelope of the $H_{1} C_{1}$ component (diamonds), and (iv) the average of the curvature of the numerical exciton dispersion weighted with the same function as (iii) (circles, the line is a guide to the eye).

The top curve displays the values for the Bohr radius that we have used for the calculation of (iii) and (iv). Arrows at either sides of the lower part of Fig. 11 mark the mass in the diagonal approximation in the well and barrier bulk materials.

Fig. 11 demonstrates the failure of the diagonal Luttinger approximation (i) to describe even the $H H_{1}$ subband curvatures at the $\Gamma$-point due to the degeneracy of heavy- and light-hole bands in the unstrained bulk. However, even the correct single-particle subband curvatures at the $\Gamma$-point (ii) fail to describe accurately the curvature of the groundstate exciton dispersion at $Q=0$. This is mainly due to the finite extension of the exciton in $k$-space that implies an averaging of the subband dispersions over a region of approximately $1 / a_{B}$ near the $\Gamma$-point and partly to the Coulomb coupling to higher subbands. Both effects tend to make the groundstate exciton heavier. The mass derived from the curvature of the groundstate exciton at $Q=0$ (not shown) lies between curves (ii) and (iii).

The numerically obtained "best" mass values (iv) show a quantitatively and qualitatively different behavior from curves (i) and (ii). For very narrow QW the subbands become flatter at the $\Gamma$-point because of the larger penetration into the barriers where the exciton becomes again heavier, as in models (i), (ii). But, for large $L$ the region of the $H H_{1}-L H_{1}$ avoided crossing comes to a distance of approximately $1 / a_{B}$ to the $\Gamma$-point and the exciton, averaging over the flatter subband dispersion, becomes heavier.

The quality of our semi-analytical result for the average exciton mass (iii) has to be judged according to its deviation from the numerical average (iv). The non-monotonous behavior is clearly seen for the mass (iii) obtained using only the $H_{1}$ subband dispersion and the fitted Bohr radius. The mass values (iii) are somewhat smaller $(<10 \%)$ than the ones of curve (iv). This is due to the enhancement of the binding energy for $Q \neq 0$ that yields larger average masses (iv) than one would expect based on the one-particle subband dispersions and the $Q=0$ groundstate exciton. Our semi-analytical average groundstate exciton mass (iii) reaches a minimum approximately at the QW width where the maximum binding energy is reached.

The small differences between curves (iii) and (iv) demonstrate the quality of our expression (25:26) for the average exciton groundstate mass. For this reasonable and easy-to-use mass expression, only a good estimate for the in-plane Bohr radius and the dispersion of the involved single-particle subbands is needed.

In the paper by Triques and Brum [18], average exciton effective masses were calculated that are relevant to the formation process of excitons in two different scenarios. These masses are defined by parabolic fits within a relevant energy range of: (a) $5 \mathrm{meV}$, approximately half the exciton binding energy, in the case that the particles first relax and then form an exciton of kinetic energy lower than the binding energy and (b) $36 \mathrm{meV}$ in the case that the exciton is formed very fast and relaxes initially via optical phonon emission, reaching energies below $36 \mathrm{meV}$, the energy 
of the GaAs-LO phonon. These energy ranges translate to $Q$ values in general much larger than $1 / a_{B}$. Therefore, these average masses should be larger than those of the present work. However, the values published in [18] for the $5 \mathrm{meV}$ mass $\left(0.2 m_{0} \leq M_{g}^{X} \leq 0.3 m_{0}\right.$ for $\left.L \leq 10 \mathrm{~nm}\right)$ are smaller than ours for narrow QW. Our average masses of scenario (a) are not falling below $0.3 m_{0}$ and show a smooth minimum for a $\mathrm{QW}$ of width somewhere between $2 \mathrm{~nm}$ and $5 \mathrm{~nm}$. This difference can be traced back mainly to the inefficiency for narrow QW of their method involving an expansion in the subband states at the $\Gamma$-point, as already remarked by the authors themselves. Indeed, for narrow QW the few states at the $\Gamma$-point can not provide the needed flexibility to simulate states far away from the $\Gamma$-point. This is substantiated by the lower left panel in Fig. 8 showing the groundstate exciton at $Q=0.5 \mathrm{~nm}^{-1}$ for the $5 \mathrm{~nm}$ QW. The $z_{e} z_{h}$-plots show a substantial $m_{J}=+\frac{1}{2}$ spin component. In an expansion of the $z_{h}$ dependence in the hole subband states at the $\Gamma$-point this component would need a $\mathrm{LH}_{2}$ subband to be described efficiently. However, for the $5 \mathrm{~nm}$ QW only the $H H_{1}, L H_{1}, H H_{2}$ subbands exist below the top of the barrier.

In Fig. 5(c), we compare the results of Ref. [18] with ours for the $5 \mathrm{~nm} \mathrm{QW.} \mathrm{For} \mathrm{this} \mathrm{narrow} \mathrm{QW,} \mathrm{their} \mathrm{exciton}$ and $H H_{1}$ subband dispersions are much steeper than ours. The discrepancy is not due to the different parameters as a comparison with the subband dispersion calculated exactly with the transfer matrix method for the parameters of Ref. [18] demonstrates (diamonds).

We remarked already, that different parts of the non-parabolic groundstate exciton dispersion are relevant for different physical process, and we gave exciton localization [1, 1 ] and cooling of a non-thermal exciton population [18] as examples. We would like to discuss two more possible experimental consequences of anharmonicities using Fig. 1 as illustration. First, an exciton population loosing energy by acoustic phonon emission at low temperatures could experience a bottle-neck effect, i.e., an increased population of the $k$-space region near $0.15 \mathrm{~nm}^{-1}$. Scattering closer towards the $\Gamma$-point will be suppressed by the decreased density of final states. A second, more directly observable consequence is the temperature dependence of the exciton lifetime. Evaluation of the latter along the lines of Ref. 34] with the groundstate dispersion of Fig. 1 yields a super-linear increase of the exciton lifetime with temperature: The slope increases in the range from 5 to $20 \mathrm{~K}$ by a factor 2.2 (not shown).

\section{CONCLUDING REMARKS}

In summary, we performed $\vec{k} \cdot \vec{p}$ multiband exciton dispersion calculations of high accuracy even for very large COM momentum and narrow QW using the well known expansion in subband states. For the high quality of the numerical results, an optimized COM transformation was essential. The optimized COM transformation is related to an average exciton kinetic mass for which a simple semi-analytical expression is derived. This eliminates for many practical purposes the need to actually calculate the groundstate dispersion. The groundstate exciton dispersion is found to follow closely the respective exciton continuum edge.

In addition, we demonstrated that multiband groundstate exciton dispersion calculations are feasible in real space using a finite-differences scheme. Besides the essential optimized COM transformation, a convenient groundstateadapted discretization of the Coulomb potential enhances numerical accuracy. This method promises to give results for systems where the first one is not practicable. Its generalization for finite-element schemes is straightforward.

\section{ACKNOWLEDGMENTS}

This work has been funded by the Deutsche Forschungsgemeinschaft in the frame of SFB 296. We wish also to thank the Rechenzentrum of the Humboldt University and the Konrad-Zuse-Zentrum in Berlin for their support and access to their Cray J932 (project bvph08as).

\section{APPENDIX: DISCRETIZATION OF THE COULOMB POTENTIAL}

In order to attain a simple discretization for the interaction of some Hamilton operator, Glutsch, Chemla, and Bechstedt [30] proposed to discretize on the same mesh another operator whose groundstate is analytically known with the same interaction but with a simple kinetic term (mass $m^{\text {ref }}$ ). We will call this the reference system. For illustration, consider a simple one-dimensional system with known groundstate wavefunction $g(x)$ of energy $E_{g}^{r e f}$. The Schrödinger equation of the reference system discretized on a mesh $x_{i}=i \cdot \Delta_{x}$ reads

$$
-\frac{\hbar^{2}}{2 m^{r e f} \Delta_{x}^{2}}\left(g\left(x_{i+1}\right)-2 g\left(x_{i}\right)+g\left(x_{i-1}\right)\right)+V\left(x_{i}\right) g\left(x_{i}\right)=E_{g}^{r e f} g\left(x_{i}\right)
$$


and yields the groundstate adapted discretization

$$
V\left(x_{i}\right)=E_{g}^{r e f}+\frac{\hbar^{2}}{2 m^{r e f} \Delta_{x}^{2}} \frac{g\left(x_{i+1}\right)-2 g\left(x_{i}\right)+g\left(x_{i-1}\right)}{g\left(x_{i}\right)}, g\left(x_{i}\right) \neq 0 .
$$

This procedure is very simple, easy to implement, cheap to calculate, and gives for the reference system always the correct groundstate energy, regardless how inappropriate the mesh is. In addition, no special handling for potentials with integrable singularities is needed.
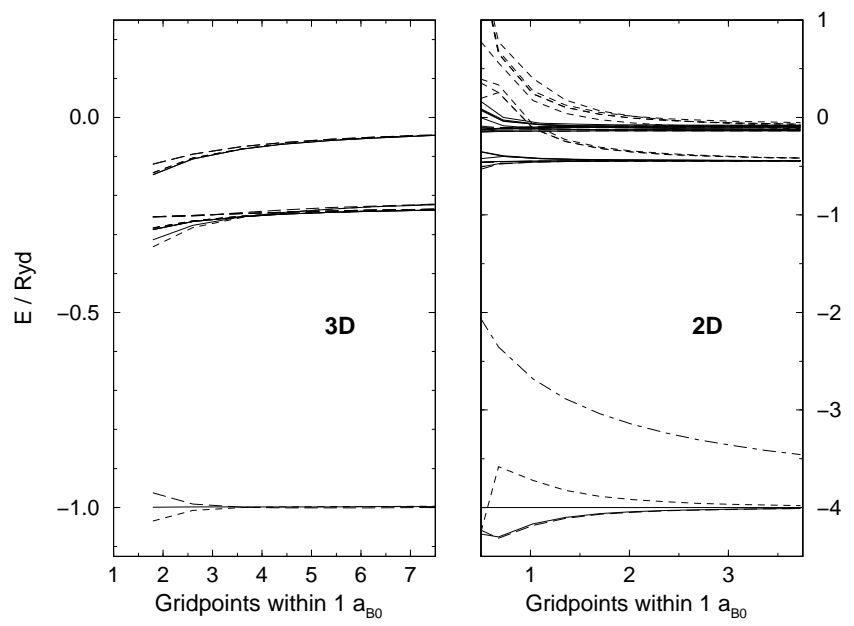

FIG. 12. The lowest eigenvalues calculated in real space of the ideal 3D exciton (left) and 2D exciton (right) of Bohr radius $a_{B 0}$ plotted versus grid density. Discretization of the Coulomb potential as described in the Appendix with reference Bohr radius $a_{B}^{r e f}: a_{B 0}$ (thick), $2 a_{B 0}$ (dashed), $a_{B 0} / 2$ (long dashed). For the $2 \mathrm{D}$ case, also results are shown for the groundstate with the potential integrated analytically in every mesh cell, Eq. (31), (thin) and for the groundstate with the "naive discretization" (dot-dashed). The former curve is hardly to distinguish from the one for $a_{B}^{r e f}=2 a_{B 0}$.

Using this discretization for a reference state similar enough to the one sought, one can expect good convergence with mesh size. In order to check how the dependence of the potential discretization on the reference state influences the results, we performed calculations of the ideal 2D and 3D excitons using discretizations of the Coulomb potential based on reference excitonic groundstates with various Bohr radii. Fig. 12 shows the lowest numerical eigenvalues as a function of gridpoint density. One can see that the correct estimation of the "unknown" groundstate is not so critical: a reference Bohr radius $a_{B}$ within a factor of two from the actual one, $a_{B 0}$, still gives good results for the groundstate for reasonable mesh densities. Apparently, especially for the $2 \mathrm{D}$ case, it is better to choose $a_{B}$ rather larger than smaller in order to get good results also for the excited states.

We also show for the $2 \mathrm{D}$ case the results obtained with the potential integrated analytically on each Cartesian mesh element, using

$$
\iint \frac{d x d y}{r}=y \ln \left(x+\sqrt{x^{2}+y^{2}}\right)+x \ln \left(y+\sqrt{x^{2}+y^{2}}\right) .
$$

This gives also a very good convergence with the mesh size. The $2 \mathrm{D}$ result obtained for the groundstate with the potential integrated analytically only at the origin, and taking everywhere else its value at each gridpoint ('naive' discretization) is included in Fig. 12. Since both discretizations are the same at the origin the difference does not originate from the divergence at this point. The superiority of the discretizations (30) and (31) is obvious. We therefore expect Eq. (30) to yield good results even with not very dense meshes.

For the 3D case a similar expression to Eq. (31) for the Coulomb potential integrated analytically on a rectangular box can be derived. However, we could not use this result to attain an alternative discretization for the real-space QW calculations because the natural mesh $\left(\rho_{x}, \rho_{y}, z_{e}, z_{h}\right)$ is not Cartesian in the relative coordinate $z_{e}-z_{h}$. 
[1] E. Runge and R. Zimmermann, Festkörperprobleme/Advances in Solid State Physics 38, 251 (1998); R. Zimmermann and E. Runge, phys. stat. sol. (a) 164, 511 (1997)

[2] R. Kumar, A. S. Vengurlekar, S. S. Prabhu, J. Shah, and L. N. Pfeiffer, Phys. Rev. B 54, 4891 (1996); B. Deveaud, F. Clérot, N. Roy, K. Satzke, B. Sermage, and D. S. Katzer, Phys. Rev. Lett. 67, 2355 (1991); T. C. Damen, J. Shah, D. Y. Oberli, D. S. Chemla, J. E. Cunningham, and J. M. Kuo, Phys. Rev. B 42, 7434 (1990); Ph. Roussignol, C. Delalande, A. Vinattieri, L. Carraresi, and M. Colocci, Phys. Rev. B 45, 6965 (1992)

[3] M. Z. Maialle, E. A. Andrada e Silva, and L. J. Sham, Phys. Rev. B 47, 15776 (1994); A. Vinattieri, J. Shah, T. C. Damen, D. S. Kim, L. N. Pfeiffer, M. Z. Maialle and L. J. Sham, Phys. Rev. B 50, 10868 (1994); J. Puls, F. Henneberger, M. Rabe, and A. Siarkos, J. of Cryst. Growth 184/185, 787 (1998)

[4] H. F. Hess, E. Betzig, T. D. Harris, L. N. Pfeiffer, and K. W. West, Science 264, 1740 (1994)

[5] G. Dresselhaus, J. Phys. Chem. Solids 1, 14 (1956)

[6] M. Altarelli and N. O. Lipari, Phys. Rev. B 15, 4898 (1977)

[7] H. Chu, G. D. Sanders, and Y.-C. Chang, Phys. Rev. B 36, 7955 (1987)

[8] P. Michler, A. Hangleiter, A. Moritz, G. Fuchs, V. Härle, and F. Scholz, Phys. Rev. B 48, 11991 (1993)

[9] D. A. Broido and L. J. Sham, Phys. Rev. B 31, 888 (1985)

[10] D. A. Broido and L. J. Sham, Phys. Rev. B 34, 3917 (1986)

[11] G. D Sanders and Y.-C. Chang, Phys. Rev. B 35, 1300 (1987)

[12] L. C. Andreani and A. Pasquarello, Phys. Rev. B 42, 8928 (1990)

[13] C. Y.-P. Chao and S. L. Chuang, Phys. Rev. B 48, 8210 (1992)

[14] B. Zhu and Y.-C. Chang, Phys. Rev. B 50, 11932 (1994)

[15] J. Chen, R. Chen, and K. K. Bajaj, Phys. Rev. B 50, 10947 (1994)

[16] R. Winkler, Phys. Rev. B 51, 14395 (1995)

[17] S. Jorda, U. Rössler, and D. Broido, Phys. Rev. B 48, 1669 (1993)

[18] A. L. C. Triques and J. A. Brum, Phys. Rev. B 56, 2094 (1997)

[19] A. Siarkos, E. Runge, and R. Zimmermann, Annalen der Physik (Leipzig) 7, 523 (1998)

[20] E. Kapon, D. M. Hwang, and R. Bhat, Phys. Rev. Lett. 63, 430 (1989); O. Stier and D. Bimberg, Phys. Rev. B 55, 7726 (1997)

[21] J. M. Luttinger, Phys. Rev. B 102, 1030 (1956)

[22] B. Zhu and K. Huang, Phys. Rev. B 36, 8102 (1987)

[23] D. B. Tran Thoai, R. Zimmermann, M. Grundmann, and D. Bimberg, Phys. Rev. B 42, 5906 (1990)

[24] E. L. Ivchenko, A, Y. Kaminski, and U. Rössler, Phys. Rev. B 54, 5852 (1996); T. Hassenkam, S. Pedersen, K. Baklanov, A. Kristensen, C. B. Sorensen, P. E. Lindelof, F. G. Pikus, and G. E. Pikus, Phys. Rev. B 55, 9298 (1996); R. Winkler and U. Rössler, Phys. Rev. B 48, 8919 (1993)

[25] L. C. Andreani, A. Pasquarello, and F. Bassani, Phys. Rev. B 36, 5887 (1987)

[26] M. A. Kanehisa, Physica 117B \& 118B, 275 (1983)

[27] L. R. Ram-Mohan, K. H. Yoo, and R. L. Aggarwal, Phys. Rev. B 38, 6151 (1988)

[28] ARPACK and the parallel version PARPACK can be downloaded free of charge at http://www.caam.rice.edu/software

[29] U. Elsner, V. Mehrmann, F. Milde, R.A. Römer, and M. Schreiber, SIAM J. Sci. Comp 20, 2089 (1999)

[30] S. Glutsch, D. S. Chemla, and F. Bechstedt, Phys. Rev. B 54, 11592 (1996)

[31] Unfortunately, in Fig. 1 of Ref. [19] a different convention was used, with $\beta$ replaced by $\beta-1 / 2$.

[32] Landolt-Bornstein, New Series, Part III/2a, edited by O. Madelung, Springer, Berlin, 1982; A. Onton, Festkörperprobleme XIII, 59 (1973); E. Hess, I. Topol, K.-R. Schulze, H. Neumann, and K. Unger, phys. stat. sol. (b) 55, 187 (1973)

[33] B. A. Foreman, Phys. Rev. B 49, 1757 (1994)

[34] L. C. Andreani, in Confined Electrons and Photons, edited by E. Burstein and C. Weisbuch, Nato ASI Series Vol. 349, Plenum, New York, 1995, p. 57-112 